\title{
The Determination of the Physico-Chemical Properties of Nanoemulsion
}

\author{
Maurice O. Iwunze \\ Department of Chemistry, Morgan State University, MD 21251, USA
}

\begin{abstract}
Nanoemulsion is a heterogeneous fluid system that has been used in chemistry, biology and industry as a reaction medium and in medicine and allied sciences as a delivery vehicle for poorly water-insoluble biochemical compounds. It is made up of water-oil-surfactant and co-surfactant (usually a short chain alcohol). This fluid system has come of age and it is necessary that its physico-chemical properties be properly determined and documented. Such physico-chemical properties include dielectric constant, polarity, refractive index, viscosity, acidity, basicity and density. These properties are very important in designing a fruitful, effective and meaningful interpretation of chemical reactions and its utility. These are determined and documented in this work.
\end{abstract}

Key words: Nanoemulsion, effective dielectric constant, viscosity, reihardt dye, alcohols, surfactant.

\section{Introduction}

Nanoemulsion, (miniemulsion, as it is sometimes called) [1-4] is a heterogeneous system of oil-in-water dispersed by an amphiphilic compound (surfactant) and a co-surfactant, usually a short-chain alcohol. The mean oil droplet diameter of nanoemulsion ranges from 50 to 200 nanometers [5-17]. This heterogeneous system is known to exhibit unique properties $[18,19]$ and is used in all areas of science and technology: Its application includes solubilization of water-insoluble or poorly insoluble molecules [20-24], drug delivery $[2,6,7,9,13,20-25]$, pharmaceutical formulation [8, 9, 24], dermatology [11, 25-27] and a host of other applications. Many techniques have been used in producing a very kinetically stable nanoemulsion. Such techniques have varied from very sophisticated mechanical process [6] to ultrasonic room temperature $[9,14,28]$, including Phase Inversion Temperature (PIT) Technique [14-17, 29]. The literature is replete of reviews $[2,5,7,9,10,12,13,19,21,22,25,26]$ detailing the formulation and usage of this unique system whose physico-chemical properties have not been adequately documented. It is the objective of this

Corresponding author: Maurice O. Iwunze, professor, research fields: physical chemistry and analytical chemistry. work, therefore, to obtain and document the useful physico-chemical parameters of this unique system to aid and to enhance in its use in efficient design of chemical reactions tailored for specific in application.

\section{Experimental}

\subsection{Chemicals}

Analytical reagent grade methanol and ethanol were obtained from Pharmco. Other alcohols (propanol, butanol and hexanol) were obtained from Sigma Aldrich in $95-98 \%$ purity. Reichardt dye $\mathrm{E}_{\mathrm{T}}(30)$ of $90 \%$ purity, tetradecane and 1-pentanol of $99 \%$ purity and cetylhexadecyltrimethylammonium bromide (CTAB) were obtained from Across Chemicals. All chemicals were used as received.

\subsection{Nanoemulsion Preparation}

Appropriate weight of $\mathrm{CTAB}$ and a measured volume of water were mixed together. To this mixture a known volume of n-tetradecane as oil was added with vigorous stirring. A calculated volume of co-surfactant (1-pentanol) was added drop-wise with vigorous mechanical stirring for about five minutes before transferring it to ultrasonic sonicator bath for about 7-10 more minutes. The solution so prepared 
was isotonic, clear and translucent. This solution was stable for a considerable length of time. The Table 1 shows the composition the compounds used in this work and their values in the make-up of the nanoemulsion described in this work.

\subsection{Instruments}

The instruments used in this work include Brookfield viscometer Model RDVD II + for the viscosity determination; the VWR Conductance meter for the measurement of the solution conductance, the Abbe Digital refractometer for the determination of the refractive index; the digital PAAR density meter, model DMA 35 for density measurement and the Carry spectrophotometer model $1 \mathrm{E}$ for determination of the absorbance of the alcohols.

\section{Results and Discussion}

We show in Fig. 1 the SEM image of the $76 \%$ nanoemulsion prepared as discussed above.

The image was further analyzed for particle size distribution using the Image $\mathrm{J}$ program from Dune

Table 1 Chemicals used in the preparation of $76 \%$ nanoemulsion.

\begin{tabular}{llll}
\hline Component & Weight, g & Volume, $\mathrm{mL}$ & Percentage, \% \\
\hline Water & 174.0 & 174.0 & 76.0 \\
$\begin{array}{l}\text { CTAB } \\
\text { (Surfactant) }\end{array}$ & 12.0 & 12.63 & 5.0 \\
$\begin{array}{l}\text { Oil (tetradecane) } \\
\begin{array}{l}\text { Co-surfactant } \\
\text { (n-pentanol) }\end{array}\end{array}$ & 29.0 & 18.25 & 6.0 \\
\hline
\end{tabular}

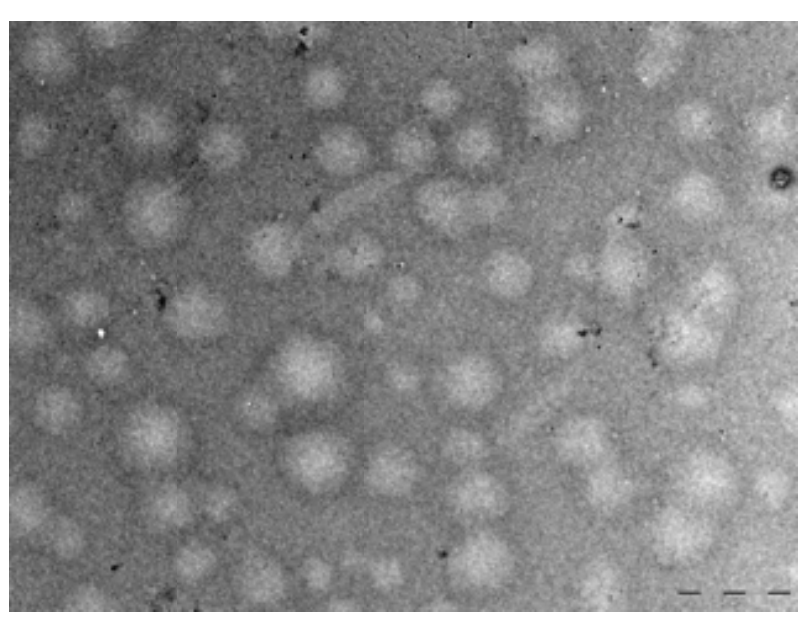

Fig. 1 SEM image of $76 \%$ nanoemulsion.
Sciences, Inc. [30]. The plot for this distribution is shown in Fig. 2. It can be seen that the plot peaked at 123.0 nanometers.

We used this value as the average diameter of the oil droplet in this preparation.

Reichardt dye (RD) also known as ET [30] (2, 6-Diphenyl-4-(2,4,6-triphenyl-1-pyridino)phenolate) is often used for polarity evaluation of solvents. This is because this compound is very sensitive to its environment. Its maximum wavelength shifts in accordance with the polarity of a given solvent. Fig. 3 is the chemical structure of this compound.

The equation that is often used to calculate the $\mathrm{E}_{\mathrm{T}}$ (30) values is

$$
\mathrm{E}_{\mathrm{T}}(30), \mathrm{Kcal} / \mathrm{mol}=28591 / \lambda_{\max }, \mathrm{nm}
$$

$\lambda_{\max }$ in this equation is a characteristic maximum wavelength of $\mathrm{E}_{\mathrm{T}}(30)$ in a given solvent.

Using the solvatochromic technique developed by Reichardt, Taft and other workers [31-37] the $\mathrm{E}_{\mathrm{T}}$ (30) value of the alcohols used in this work (methanol through hexanol) were obtained in the $76 \%$ nanoemulsion. We show in Fig. 4 a typical absorbance of $\mathrm{Rd}$ solubilized in propanol and in the prepared nanoemulsion. A closer look will reveal that the maximum wavelength of the Reichardt's dye solubilized in 1-propanol is slightly shifted bathochromically compared to the RD solubilized in the $76 \%$ nanoemulsion. However, it was noticed that the observed shift is characteristic for all the alcohols

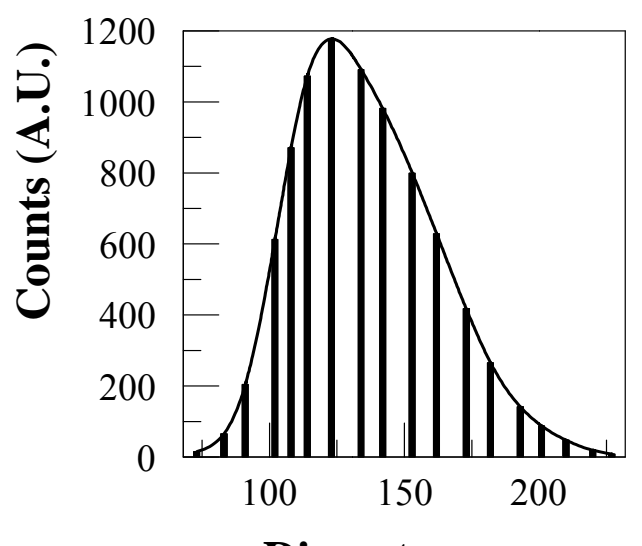

Diameter, nm

Fig. 2 Plot of the Size Distributon of Oil Droplet in 76\% Emulsion. 
<smiles></smiles>

Fig. 3 Structure of Reichardt's Dye $\left(\mathrm{E}_{\mathrm{T}}(\mathbf{3 0})\right.$.

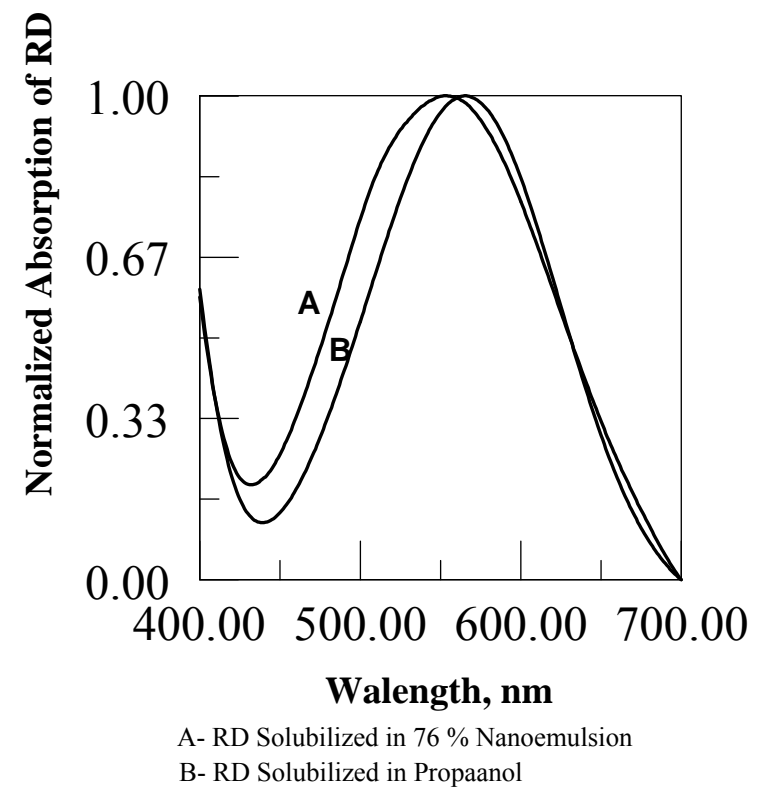

Fig. 4 Plot of the absorbance spectrum of $\mathrm{RD}$ in $\mathbf{7 6 \%}$ nanoemu and in propanol.

used. This observed shift is seen to be a function of the respective dielectric constant of a given alcohol. Table 2 gives the $\mathrm{E}_{\mathrm{T}}(30)$ of the alcohols and their respective dielectric constants The values of the dielectric constants $(\varepsilon)$ used were obtained from the Minnesota Solvent Descriptor Database [38].

When the observed $\mathrm{E}_{\mathrm{T}}(30)$ values in $76 \%$ nanoemulsion are plotted as a function of the dielectric constants of the alcohols, a straight line curve is obtained as can be seen in Fig. 5.

The slope of this curve is 0.3356 and the intercept is 44.1086 with a correlation coefficient of $99.70 \%$. Using these values the regression equation obtained is:

$$
\mathrm{E}_{\mathrm{T}}(30)=0.3356 \varepsilon+44.1086
$$

From this equation the effective dielectric constant for each alcohol in $\mathrm{E}_{\mathrm{T}}$ (30) is determined with the equation:

$$
\varepsilon_{\mathrm{f}}=\left(\mathrm{E}_{\mathrm{T}}(30)-44.1086\right) / 0.3356
$$

In Eq. (3), $\varepsilon_{\mathrm{f}}$ stands for effective dielectric constant as defined by Zachariasse and his co-workers [39]. The $E_{T}(30)$ is also a function of the polarity of the fluid system. We have used the Bakshiev equation $\mathrm{F}_{2}$ $(\varepsilon, n)[40]$ in this case to show that the observed $\mathrm{E}_{\mathrm{T}}$ (30) is also linear with the relative polarity of some organic solvent as can be seen in Fig. 6 .

Table 2 The calculated $\mathrm{E}_{\mathrm{T}}$ (30) values of the alcohols and the dielectric constants.

\begin{tabular}{llll}
\hline $\begin{array}{l}\text { Solvent } \\
\text { number }\end{array}$ & Solvent & $\begin{array}{l}\text { Dielectric } \\
\text { constant }(\varepsilon)\end{array}$ & $\begin{array}{l}\mathrm{E}_{\mathrm{T}}(30), \\
\mathrm{Kcal} / \mathrm{mol}\end{array}$ \\
\hline 1 & methanol & 31.613 & 55.09 \\
2 & ethanol & 24.852 & 52.61 \\
3 & propanol & 20.521 & 50.69 \\
4 & butanol & 17.332 & 49.81 \\
5 & pentanol & 15.13 & 49.21 \\
6 & hexanol & 12.51 & 48.79 \\
\hline
\end{tabular}

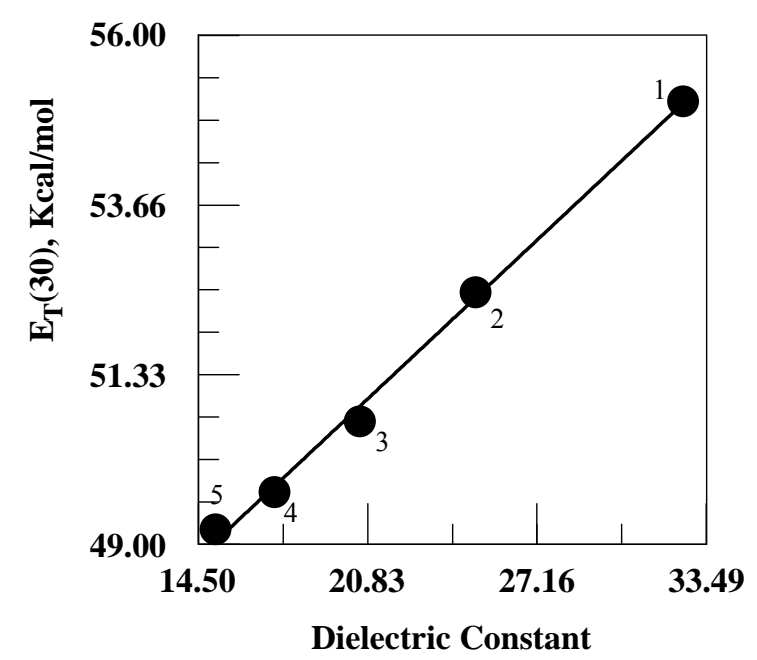

Fig. 5 Plot of ET(30) versus dielectric constants of alcohols. 


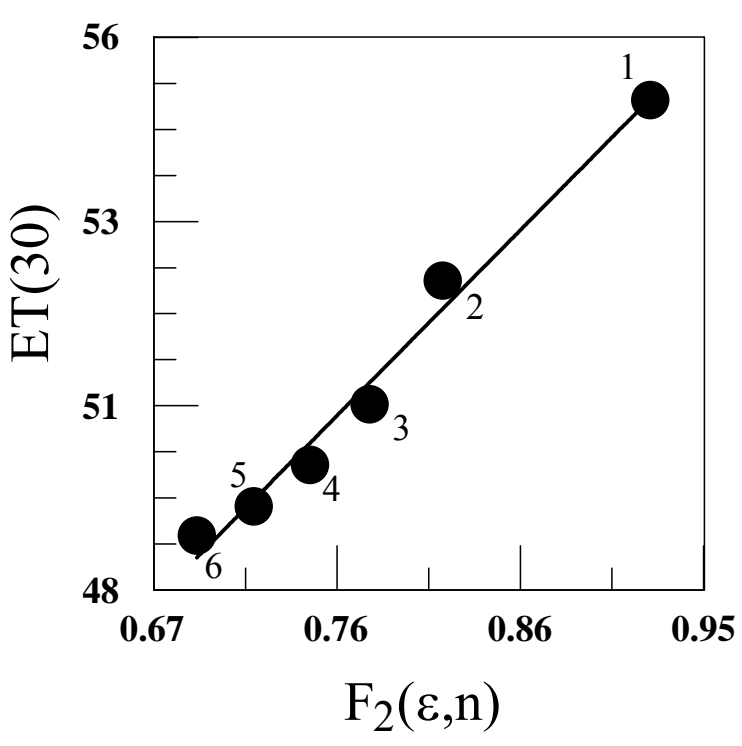

Fig. 6 Plot of $\mathrm{E}_{\mathrm{T}}(30)$ versus polarity index.

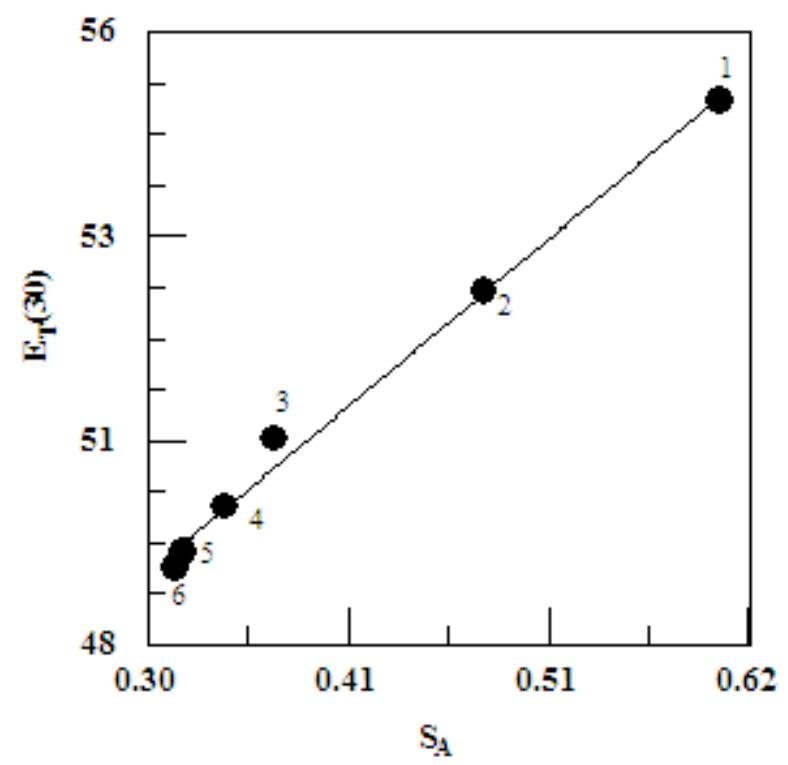

Fig. 7 Plot of $\mathrm{E}_{\mathrm{T}}(\mathbf{3 0})$ versus acidity parameter.

The $\mathrm{F}_{2}(\varepsilon, n)$ is $\mathrm{F}_{2}=\left[\frac{\varepsilon-1}{\varepsilon+2}-\frac{\mathrm{n}^{2}-1}{\mathrm{n}^{2}+2}\right]\left(\frac{2 \mathrm{n}^{2}+1}{\mathrm{n}^{2}+2}\right)$

Catalan et al. [41-46] in a series of studies showed the basicity and acidity of solvents. Using their methodology, the acidity $\left(\mathrm{S}_{\mathrm{A}}\right)$ and basicity $\left(\mathrm{S}_{\mathrm{B}}\right)$ of the prepared $76 \%$ nanoemulsion were determined. It was observed that these two parameters are also linear with the $\mathrm{E}_{\mathrm{T}}(30)$ value. The plots that show this linearity are given in Figs. 7 and 8.

The observed/calculated physico-chemical properties of the oil in water nanoemulsion $(76 \%)$ nanoemulsion are given in Table 3.

We also compared the observed polarity of this nanoemulsion with the solvent polarity obtained by

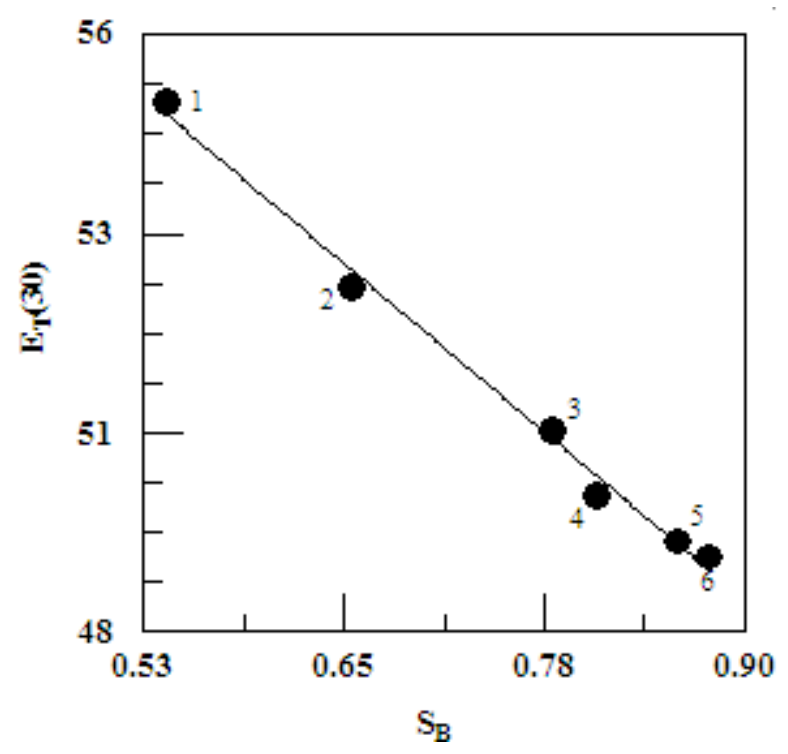

Fig. 8 Plot of $\mathrm{E}_{\mathrm{T}}(30)$ versus basicity parameter.

Table 3 The observed/calculated physico-chemical properties of $76 \%$ nanoemulsion.

\begin{tabular}{ll}
\hline Parameter & Value \\
\hline $\mathrm{E}_{\mathrm{T}}(30)$ & $51.702 \mathrm{Kcal} / \mathrm{mol}$ \\
Effective dielectric constant, $\varepsilon_{\mathrm{f}}$ & 22.59 \\
Polarity & 0.633 \\
Refractive index, $\mathrm{n}$ & 1.2631 \\
Density & $0.961 \mathrm{~g} / \mathrm{mL}$ \\
Acidity parameter, SA & 0.435 \\
Basicity parameter, SB & 0.721 \\
Conductance & $10.17 \mathrm{k \mu S}$ \\
Viscosity, $\eta$ & $16.0 \mathrm{cP}$ \\
\hline
\end{tabular}

Table 4 Comparison of the polarity of different solvents with the prepared nanoemulsion.

\begin{tabular}{ll}
\hline Compound & Polarity \\
\hline Hexane & 0.009 \\
Benzene & 0.111 \\
Chloroform & 0.259 \\
DMF & 0.385 \\
DMSO & 0.444 \\
Acetonitrile & 0.460 \\
1-Propanol & 0.617 \\
Acetic acid & 0.648 \\
Glycerin & 0.812 \\
Water & 1.000 \\
$76 \%$ nanoemulsion & 0.633 \\
\hline
\end{tabular}


other workers using different methods [47] as compiled by Prof. Steve Murov. This is given in Table 4.

It can be seen that the polarity of $76 \%$ nanoemusion prepared in this work is reasonably close to the polarity of 1-propanol as shown in Fig. 4.

\section{Conclusion}

We have shown in this work that the physico-chemical properties of nanoemulsion can be determined using $\mathrm{RD}$ and the values obtained are reasonably comparable to literature values. Also, nanoemulsion system is a very good solubilizing medium for molecules that are poorly soluble in water and is therefore usable in both pharmaceutical formulary, dermatology and a host of other applications.

\section{Acknowledgement}

The author is very grateful to the Chemistry Department of Morgan State University for the support of this work. The author is also very grateful to Prof. Michael McCaffery of the Johns Hopkins University for his help in obtaining the SEM images of the prepared nanoemulsion.

\section{References}

[1] Zhang Helgeson, M. E. 2016. "Colloidal Behavior of Nanoemulsions: Interactions, Structure and Rheology." Current Opinion in Colloid \& Interface Science 25: 39-50.

[2] Lovelyn, C., Attama, A. A., and Biomaterials, J. 2001. Nanobiotechnology 2: 626-39.

[3] Asua, J. M. 2002. Progress in Polymer Science 27: 1283-346.

[4] Landfester, K. 2001. "Polyreactions in Miniemulsions." Macromolecular Rapid Communications 22 (12): 896-936.

[5] Gupta, A., Eral, B. B., Hatton, T. A., and Doyle, P. S. 2016. Soft Matter 12: 2826-41.

[6] Jaiswal, M., Dudhe, R., and Sharma, P. K. 2015. "Nanoemulsion: An Advanced Mode of Drug Delivery System." 3Biotech. 5 (2): 123-7.

[7] Setya, S., Talegaonkar, S., and Razdan, B. K. 2014. "Nanoemulsions: Formulation Methods and Stability
Aspects." World Journal of Pharmacy and Pharmaceutical Sciences 3 (2): 2214-28.

[8] Hamon, T. M., and Huang, J. 2014. Drug Development (October): 1-5.

[9] Solans, C., Esquena J., Forgirini, A. M., Uson, N., Morales, D., Izquierdo, P., Azemar, N., and Garcia-Celma, M. J. In Adsorption and Aggregation of Surfactants in Solution, edited by Mittal, K. L., and Dinesh, O. S. Marcel Dekker, inc. Chapter 25.

[10] Wu, Y., Li, Y. H., Gao, X. H., and Chen, H. D. 2013. "The Application of Nanoemulsion in Dermatology: An Overview." J. of Drug Targeting 21 (4): 321-7.

[11] Delmas, T., Piraux, H., Couffin, A. C., Texier, I., Vinet, F., Poulin, P., Cates, M. E., Bibette, J. 2011. "How to Prepare and Stabilize Very Small Nanoemulsions." Langmuir 27 (5): 1683-92.

[12] Rajalakshmi, R., Mahesh, K., and Kumar, C. K. A. 2011. "A Critical Review on Nano Emulsions." International Journal of Innovative Drug Discovery 1 (1): 1-8.

[13] Solans, C., Izquierdo, P., Nolla, J., Azemar, N., and Garcia-Celma, M. J. 2005. "Nano-Emulsions." Current Opinions in Collid and Interface Science 10: 102-10.

[14] Yang, H. J., Cho, W. G., and Park, S. N. 2009. "Stability of Oil-in-Water Nano-Emulsions Prepared Using the Phase Inversion Composition Method." J. Industrial and Engineering Chemistry 15: 331-5.

[15] Fernandez, P., Andre, V., Rieger, J., and Kuhnle, A. 2004 "Colloids and Surfaces A: Nano-emulsion Formation by Emulsion Phase Inversion.” Physico. Chem. Eng. Aspects 251: 53-8.

[16] Tadros, T., Izquierdo, P. Esquena, J., and Solans, C. 2004. "Formation and Stability of Nanoemulsions." Advances in Colloid and Interface Science 108-109: 303-18.

[17] Izquierdo, P., Esquena, J., Tadros, Th. F., Dederen, C., Garcia, M. J., Azemar, N., and Solans, C. 2002. "Formation and Stability of Nano-Emulsions Prepared Using the Phase Inversion Temperature Method." Langmuir 18 (1): 26-30.

[18] Gutierrez, J. M., Gonzalez, C., Maestro, A., Sole, I., Pey, C. M., and Nolla, J. 2008. "Nano-emulsions: New Applications and Optimization of Their Preparation." Current Opinions in Colloid and Interface Science 13: 245-51.

[19] Mason, T. G., Wilking, J. N., Meleson, K., Chang, C. B., and Graves, S. M. 2016. "Nanoemulsions: Formation, Structure, and Physical Properties." J. Phys: Condensed Matter 18: R635-66.

[20] Zhang,Y., Shang, Z., Gao, C. G., Du, M., Xu. S., Song, H., and Liu, T. 2014. "Nanoemulsion for Solubilization, Stabilization, and in Vitro Release of Pterostilbene for Oral Delivery." AAPS Pharm Sci Tech. 15 (4), 1000-8.

[21] Thakur, A., Walia, M. K., and Kumar, S. L. 2013. "In 
Enhancement of Bioavailability of Poorly Soluble Drugs: A Review." H. Pharmacophore 4 (1): 15-25.

[22] Debnath, S., and Satayanarayana, Kumar, V. 2001. An International Journal of Advances in Pharmaceutical Sciences 2 (2-3): 72-82.

[23] Shakeel, F., and Faisal, M. S. 2010. "Nano-emulsion: A Promising Tool for Solubility and Dissolution Enhancement Of Celecoxib." Pharm. Dev. Technol. 15 (1): 53-6.

[24] Tiwari, S. B., Shenoy, D. B., and Amiji, M. M. 2006. NSTI-Nanotech. 1: 475-8.

[25] Vadlapudi, A. D., and Mitra, A. K. 2013. "Nanomicelles: An Emerging Platform for Drug Delivery to the Eye." Ther Deliv. 4 (1): 1-3.

[26] Sutradhar, K. B., and Amin, Md. L. 2013. “ Nanoemulsions: Increasing Possibilities in Drug Delivery." European Journal of Nanomedicine. 5 (2): 97-110.

[27] Chime, S. A., Kenechukwu, F. C., and Attama, A. A. INTECH. "Nanoemulsions-Advances in Formulation, Characterization and Applications in Drug Delivery." http://dx..doi.org/10.5772/15371.

[28] Harwansh, R. K., Patra, K. C., Pareta, S. K. Singh, J., and Rhman, M. A. 2011. "Nanoemulsions as Vehicles for Transdermal Delivery of Glycyrrhizin.” Brazilian J. Pharmaceutical Sciences 47 (4): 769-78.

[29] Hashtjin, A. M., and Abbasi, S. J. 2015. "Optimization of Ultrasonic Emulsification Conditions for the Production of Orange Peel Essential Oil Nanoemulsions.” Food Sci. Technol. 52 (5): 2679-89.

[30] Dune Sciences Inc., www.dunesciences.com.

[31] Reichardt, C. 2004. "Pyridinium N-phenolate Betaine Dyes as Empirical Indicators of Solvent Polarity: Some New Findings." Pure Appl. Chem. 76 (10): 1903-19.

[32] Laglante, A. F., Spadi, M., and Bruno, T. J. 2000. J. Chem. Eng. Data. 45: 382-5.

[33] Lobaugh, J., and Rossky, P. J. 2000. "Solvent and Intramolecular Effects on the Absorption Spectrum of Betaine-30.” J. Phys. Chem. A 104: 899-907.

[34] Reichardt, C. 1994. "Solvatochromic Dyes as Solvent Polarity Indicators." Chem. Rev. 94: 2319-58.

[35] Kosower, E. M., Dodiuk, H., Tanizawa, K., Ottolenghi,
M., and Orbach, N. 1975. "Intramolecular donor-acceptor systems. Radiative and Non-Radiative Processes for the Exited States of 2-N-Arylamino-6-Naphthalenesulfonates." J. Am. Chem. Soc. 97 (8): 2167-77.

[36] Kamlet, M. J., and Taft, R. W. 1976. "The Solvatochromic Comparison Method. I. The Beta-Scale of Solvent Hydrogen-Bond Acceptor (HBA) Basicities." Ibid 98 (2): 377-83.

[37] Taft, R. W., and Kamlet, M. J. 1976. "The Solvatochromic Comparison Method. 2. The Alpha-Scale of Solvent Hydrogen-Bond Donor (HBD) Acidities." Journal of the American Chemical Society 98 (10): 2886-94.

[38] Winget, P., Dolney, D. M., Gisen, D. J., and Cramer, C. J. 1999. Minnesota Solvent Descriptor Database.

[39] Zachariasse, K. A., Phuc, N. V., and Kozanklowkz, B. J. 1981. "Investigation of Micelles, Micro-Emulsions, and Phospholipid-Bilayers with the Pyridinium N-Phenolbetaine ET(30), a Polarity Probe for Aqueous Interfaces." Phys. Chem. 85: 2676-83.

[40] Bakshiev, N. G. 1964. "Universal Intermolecular Interactions and Their Effect on the Position of the Electronic Spectra of Molecules in Two Component Solutions." Opt. Spektrosk. 16: 821-32.

[41] Jayabharathi, J., Ramanathan, P., Thanikachalaam, V., and Arunpandiyan, A. 2014. "Kamlet-Taft and Catalán Solvatochromism of Some $\pi$-Expanded Phenanthrimidazole Derivatives-DFT Analysis." Spectrochimica Acta Part A: Molecular and Biomolecular Spectroscopy 133: 201-6.

[42] Catalan, J. et al. http://www.uam.es/personal_pdi/scienclas/catalan/sa.htm.

[43] Catalan, $\mathrm{J}$. http://www.uam.es/personal_pdi/scienclass/catalan/sb.bt $\mathrm{m}$

[44] Catalan, J. http://www.uam.es/personal_pdi/scienclass/catalan/spp.ht $\mathrm{m}$.

[45] Catalan, J., and Diaz, C. 1997. Liebigs Ann. 1941-9.

[46] Catalan, J., Diaz, C., Lopez, V., Perez, P., de Paz, J. L. G., and Rodriguez, J. G. 1996. Ibid. 1785-94.

[47] https://www.murov.info//orgsolvents..html 